\title{
Using Various Evaluation Standards to Determine an Error Recovery Process in an Automation Plant
}

\author{
Akira Nakamura*1, Natsuki Yamanobe ${ }^{* 1}$, Ixchel Ramirez Alpizar ${ }^{* 1}$, Kensuke Harada ${ }^{* 1, * 2}$ and Yukiyasu Domae ${ }^{* 1}$ \\ ${ }^{*}$ Industrial Cyber Phsical System Research Center \\ National Institute of Advanced Industrial Science and Technology (AIST) \\ Second Annex, AIST Tokyo Waterfront, 2-4-7 Aomi, Koto-ku, Tokyo 135-0064 Japan \\ ${ }^{* 2}$ Robotic Manipulation Research Group \\ Systems Innovation Department \\ Graduate School of Engineering Science, Osaka University \\ 1-3 Machikaneyama, Toyonaka 560-8531, Japan \\ E-mail: a-nakamura@aist.go.jp,n-yamanobe@aist.go.jp,ixchel-ramirezalpizar@aist.go.jp, \\ harada@sys.es.osaka-u.ac.jp,domae.yukiyasu@aist.go.jp \\ www.aist.go.jp
}

\begin{abstract}
In an automated plant, an error is more likely to occur in difficult tasks, which are complicated in nature. Such a task is often re-executed after returning to the previous step when a large-scale error occurs. Therefore, deciding both the past step that the task should return to and the recovery planning following this return becomes important. In this study, error recovery planning considering these two factors using various evaluation standards is realized.
\end{abstract}

Keywords: error recovery, task stratification, error classification, automation plant

\section{Introduction}

Automated plants have been used for production in various industries due to which the efficiency of production increases. On the contrary, system automation also leads to errors. Therefore, the progress of the error recovery method is crucial, which has demanded the increased study of error recovery in recent years. ${ }^{1-5}$ However, the recovery technique used in these studies is ad hoc, and it is difficult to utilize them in various actual plants.

For several years, we performed a study on the systematization of the error recovery theory. We proposed a novel error recovery method based on the concepts of both task stratification and error classifications. ${ }^{6-8}$ The main part of this method consists of fundamental elements with sequences of sensing, modeling, planning, and execution (Fig. 1). If an error occurs here, the process advances to the recovery part. In this part, the error cause is estimated, error is classified, system is corrected, and process is reexecuted using the corrected system with an improved reliability.

Currently, deciding both the past step that the process should return to and the recovery planning after returning has become problematic. For this, we proposed a planning of the error recovery by deriving these two factors in consideration of cost. $^{8}$ In this study, we proposed a planning method for error recovery derived using various evaluation standards.

(C) The 2021 International Conference on Artificial Life and Robotics (ICAROB2021), January 21 to 24, 2021 


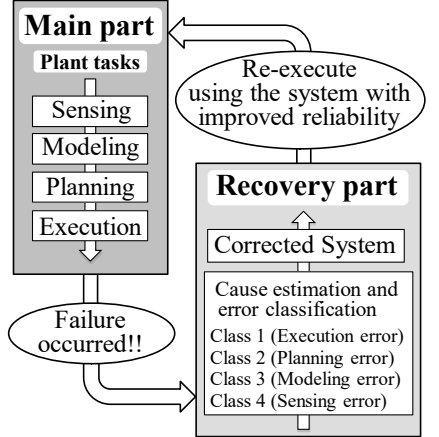

Fig. 1 Robot task system with an error recovery function
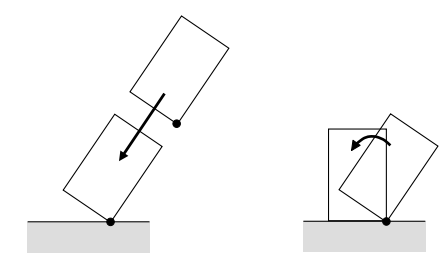

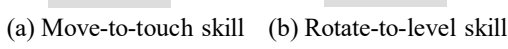

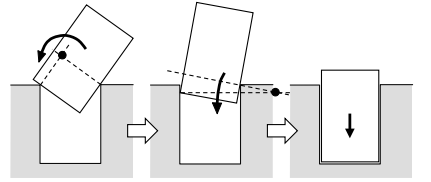

(c) Rotate-to-insert skill

Fig. 2 Three fundamental skills

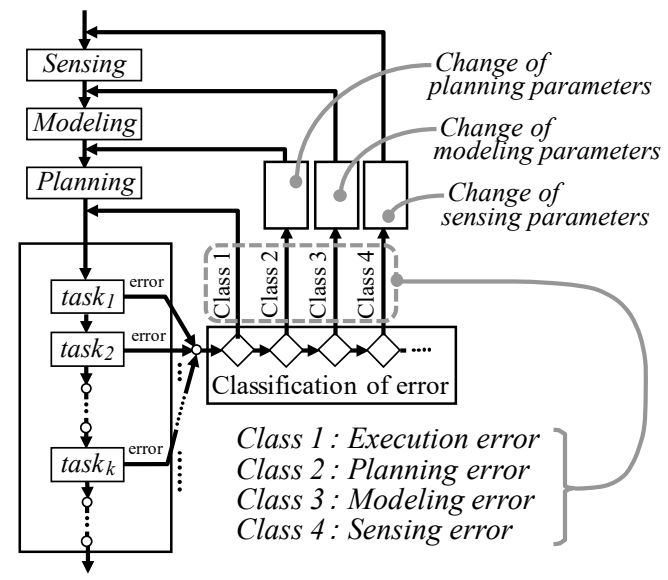

Fig. 4 Fundamental process flow with error recovery

The concept of skill, which is motion primitive, is described in Section 2. The error recovery technique is explained in Section 3. A method of recovery planning using plural evaluation standards is proposed in Section 4, and finally, a simple sample is presented in Section 5.

\section{Concept of Skill}

In this study, it is assumed that "skill" represents the unit of motion. In this section, we describe the indispensable aspects of skills, which are components of human behavior and automated plant operations. ${ }^{9-11}$

\subsection{Skill primitives}

We derived the motion primitives constituting tasks such as assembly and carrying by analyzing a person's behavior and called this motion unit "skill".9-11 Thus, three basic skills of "move-to-touch", "rotate-to-level" and "rotate-to-insert" are important (Fig. 2). The representative person's behavior can be composed of these three basic skills and other resembled skills. The unit of machine motion can be considered to be similar to the motion primitives of human behavior.

\subsection{Stratification of tasks}

In general, it is possible to regard a task performed by an equipment in an automated plant as a hierarchical structure (Fig. 3). ${ }^{9-11}$ The layer " task $^{(i+1) \text { " }}$ occurs one tier above the layer "task(i)", and the layer "skill primitive" is represented by the lowest layer " $\operatorname{task}^{(0)}$."

\section{Error Recovery}

In an actual environment unlike the ideal, errors in equipment performance often occur due to various causes. In this section, we describe the concept of error classification and our proposed technique of error recovery. ${ }^{6-8}$

\subsection{Error classification}

Errors can be classified into four groups: execution, planning, modeling, and sensing errors on the basis of possible causes (Fig. 4). ${ }^{6-8}$

\subsection{Error recovery based on classification}

When an error occurs in automated plants, its cause is first estimated, and a suitable correction derived according to this tentative cause is performed at the equipment system. Then, the executive process returns to the previous step, and the task is restarted from the step (Fig. 4). ${ }^{6-8}$ The same error has a lower probability of occurrence due to the corrected operation of the equipment. 
If a small-scale error arises, the process returns to the previous step in the lowest layer of the hierarchy (Fig. 4, Fig. 5). If an error is large-scale, the process goes back to the previous step in the high-ranking hierarchy layer. In both cases, it is restarted from the previous step (Fig. 5).

\subsection{Candidate processes for recovery}

Let us consider candidates for error recovery processes. In the previous subsection, it was shown that the step in which the process returns to after the error occurs varies according to its scale. However, it is possible to back up bigger than a retreat step of necessity minimum.

Figure 6 shows various possible recovery processes when an error occurs at subtask $m$ in task $T$ with start $S$ and goal $G$. Then, task $T$ consists of a sequence of $\operatorname{subtask}_{k}(k=1,2,3, \cdots, n)$, which comprises a skill primitive sequence [ skill $^{k}$, skill ${ }_{2}, \cdots$, skill $\left.{ }_{n \_k(\max )}\right]$, where subtask $_{m}$ indicates the minimum traceable unit explained in the study, ${ }^{6}$ that is, the smallest unit, wherein it is necessary to return to the first node of a skill primitive sequence if an error occurs. In the absence of any problem, there is a possibility that the execution of several skills will be continued within subtask $_{m}$ without returning to the previous step immediately after the error occurrence.

After the process returns to the $\mathrm{j}$-th node before subtask $_{j}(j=1,2,3, \cdots, m)$, the process [ subtask $k_{j}$ subtask $_{j+1}, \cdots$, subtask $_{n}$ ] proceeds along the center bold arrows in the corrected plant (Fig. 6). However, reexecution using the same sequence of subtasks is often impossible for various reasons such as the displacement of the target object and the change in the arrangement of objects. In such a case, an equivalent task of the original sequence, which is derived by large-scale re-planning of subtasks, is carried out as shown in the right-side arrows of Fig. 6.

\section{Evaluation Standards for Selection of a Recovery Process}

The previous section shows that there is a possibility that many recovery processes are generated. Therefore, it becomes important to restrict routes by deciding both the prior step that should be returned to and the recovery process after return.

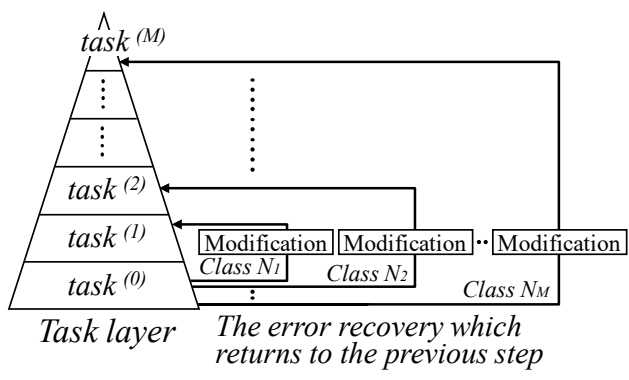

Fig. 5 The expression of task stratification and the process flow of the error recovery

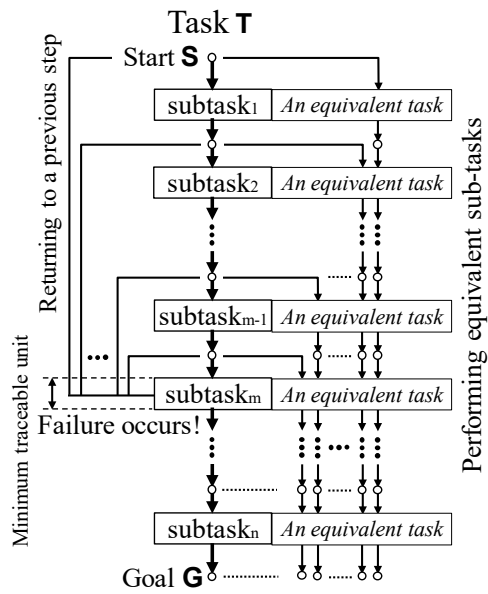

Fig. 6 Various processes of error recovery considered for a failure occurred in subtaskm

We proposed a suitable recovery planning method by considering the practical cost as an evaluation standard as in the study. ${ }^{8}$ In this study, we proposed a method to derive the most suitable recovery process using various evaluation standards.

\subsection{Evaluation standards}

Let us consider the following eight evaluation standards to select a recovery process.

(i) Cost

We considered only the cost as the evaluation standard, ${ }^{8}$ where costs include material costs, part costs, electricity bills, and planning expenses. A process with minimum practical costs is selected.

(ii) Time

Time is considered as the evaluation standard. The process where it is expected that the recovery sequence takes the shortest time is chosen.

(C) The 2021 International Conference on Artificial Life and Robotics (ICAROB2021), January 21 to 24, 2021 
(iii) Reliability

Reliability is considered as the evaluation standard. The process that emphasizes most on the operational reliability is chosen.

(iv) Safety

Safety is considered as the evaluation standard. A safety problem to a person and singular point problem are included in this standard. The process that ensures maximum safety of the operation is chosen.

\section{(v) Finishing}

The finishing of operation is considered as the evaluation standard. In the example of production and repair, a process whose outward appearance and feel are the cleanest is chosen.

(vi) Recovery data

Data on the recovery process are considered as the evaluation standard. A recovery process with a sufficient amount of data is chosen.

(vii) Tool

The tool used in the recovery process is considered as the evaluation standard. The recovery process with the special tool necessary for the practice of the work is chosen.

\section{(viii) Operator skill}

The skill of an operator in the recovery process is considered as the evaluation standard. When an expert is present, the necessary process of the skill is carried out with precedence.

\subsection{Simultaneous use of evaluation standards}

Eight types of evaluation standards were used as mentioned above, which are assumed to be used for the evaluation of the process independently. However, it is necessary to consider using several evaluation standards simultaneously considering the practical use. Of course, in this case, it is possible that the calculation of the evaluation function may become complicated.

\section{Precedence of Recovery Processes Based on Various Evaluation Standards}

In this section, the change in the priority of the recovery process based on one evaluation standard chosen among various standards discussed in Section 4 is observed via a simple sample exercise.

\subsection{Several types of recovery process}

Let us consider an assembly task in which a hook is to be stuck to the surface of a product using four precision screws, which resembles the exercise illustrated in the study. ${ }^{8}$ Figure 7 shows a sequence of this task: the tacking task is performed in the first phase (Fig. 7(a)(d)) so that these four screws are fastened temporarily to the plate part of the hook placed levelly. The erecting task is performed in the second phase (Fig. 7(e)-(f)) so that the plate part of hook with four screws stood vertically. The touching task is performed in the third phase (Fig. 7(g)-(h)) so that the hook is moved in the mounting location. The installation task is performed in the final phase (Fig. 7(i)-(m)) so that the hook is fixed on the product plane.

In this section, it is supposed that the drop of a screw occurring at the step of Fig. 7(h) represents the error as shown in Fig. 8, similar to the study. ${ }^{8}$ Let us consider the following three types of recovery sequences chosen from a lot. Please refer to this study ${ }^{8}$ for details.

\section{- $\quad$ [ER-I] Error Recovery I}

The first sequence is a recovery process based on rerun from start $\mathrm{S}$, using a new hook and four new screws, where the tacking task (Fig. 7(a)-(d)) is reexecuted using new parts (Fig. 9).

- [ER-II] Error Recovery II

The second sequence is a recovery process carried out by returning to the step of Fig. 7(d) in the tacking task, as shown in Fig. 10. Two types of methods exist for the use of a substitute of the dropped screw. One is the use of a new screw in the parts box, while the other is the reuse of the dropped screw by searching and picking up. The former is transcribed with [ER$\mathrm{II}(\mathrm{N})]$ and the latter with [ER-II(F)].

\section{- $\quad$ [ER-III] Error Recovery III}

The final sequence is a recovery process carried out by returning to a skill primitive in subtask $k_{m}$ of the failure occurrence as shown in Fig. 11. A disadvantage of this method is that the task to fasten a screw temporarily to the hole in the plate part of the hook stuck perpendicularly is more difficult than when it is placed levelly. The method uses a new screw [ER-III(N)], and the method reuses the dropped screw [ER-III(F)].

(C) The 2021 International Conference on Artificial Life and Robotics (ICAROB2021), January 21 to 24, 2021 


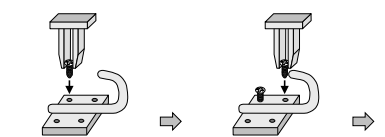

(a)

(b)

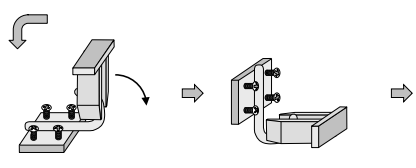

(e)

(f)

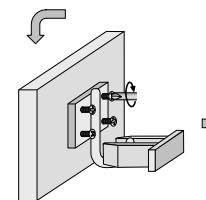

(i)

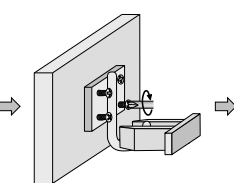

(j)

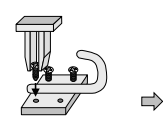

(c)

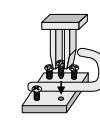

(d)

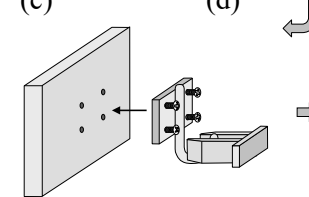

(g)

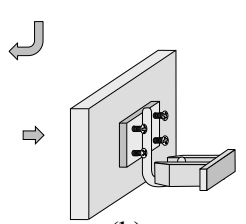

(h)

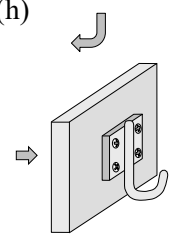

(m)

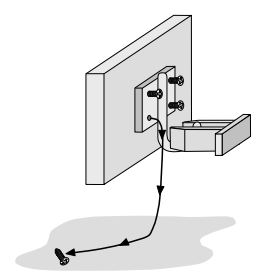

(h)'

Fig. 7 An assembly task in which a hook is stuck to a plate by four precision screws

\subsection{Suitable process in each evaluation standard}

In the case of each evaluation standard shown in Section 4 , let us consider a priority order of five recovery processes [ER-I], [ER-II(N)], [ER-II(F)], [ER-III(N)], and $[\mathrm{ER}-\mathrm{III}(\mathrm{F})]$. The explanation is carried out qualitatively, not quantitatively, for simplicity.

\section{(i) Cost}

Priority order: [ER-II(N)], [ER-III(N)], [ER-II(F)], [ER-III(F)], [ER-I].

When considering cost as the only evaluation standard, the selection method of a suitable process has already been explained. ${ }^{8}$ The costs include material costs, part costs, electricity bills, and planning expenses. [ER-II(N)] without search operation and difficulty becomes the most suitable process, and [ER-I] where many parts are exchanged become the most unsuitable one.

(ii) Time

Priority order: [ER-III(N)], [ER-II(N)], [ER-I], [ERIII(F)], [ER-II(F)].

Here, [ER-III(N)] with a small return operation and without any search operation becomes the most suitable process, while [ER-II(F)] with return and search operation becomes the most unsuitable.

(iii) Reliability

Priority order: [ER-I], [ER-II(N)], [ER-III(N)], [ERII(F)], [ER-III(F)].
[ER-I] with a restart operation becomes the most suitable process. [ER-II(N)] and [ER-III(N)] with planned operation and without search operation become the next suitable processes, whereas [ER$\mathrm{II}(\mathrm{F})]$ and [ER-III(F)] with search operation become unsuitable processes. [ER-II(N)] and [ER-II(F)] without difficult operations are suitable for each order.

\section{(iv) Safety}

Priority order: [ER-I], [ER-II(N)], [ER-II(F)], [ERIII(N)], [ER-III(F)].

[ER-I] is the most suitable process. [ER-II(N)] and $[\mathrm{ER}-\mathrm{II}(\mathrm{F})]$ without difficult operation and unstable posture become the next most suitable processes, whereas [ER-III(N)] and [ER-III(F)] with difficult operation becomes a process that is not suitable. [ER-II(N)] and [ER-III(N)] without a search operation are suitable for each order.

(v) Finishing

Priority order: Same for all.

There was no difference in finishing with respect to this task. However, the operation that may adversely affect a target item or the surroundings, such as scratch or scatter, should be avoided.

(vi) Recovery data

Priority order: A process with a large amount of data is given priority. 


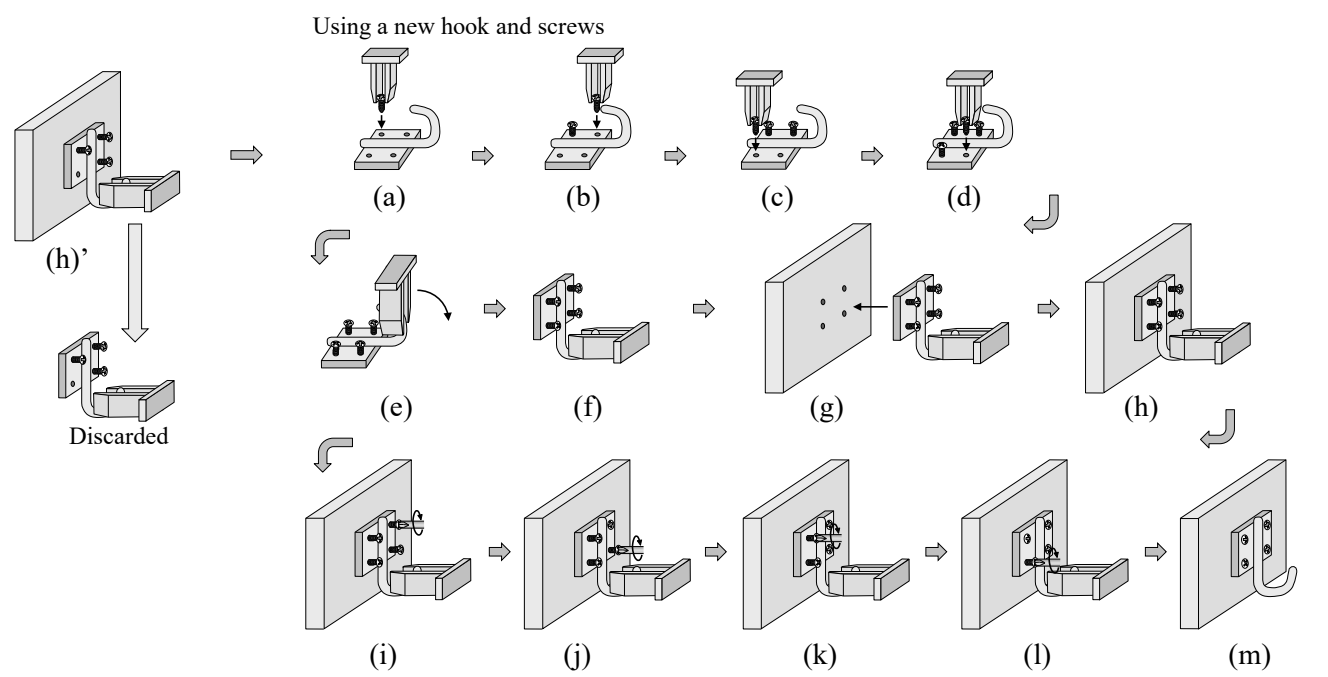

Fig. 9 [ER-I] Error Recovery I

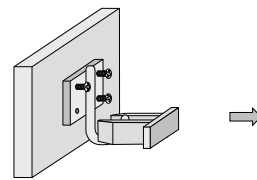

(h)'

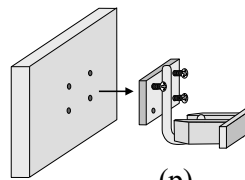

(p)

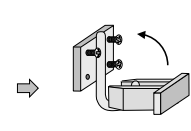

(q)

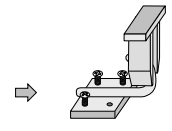

(r)

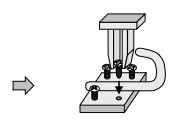

(d)

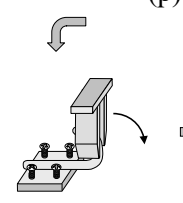

(e)

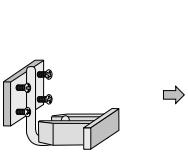

(f)

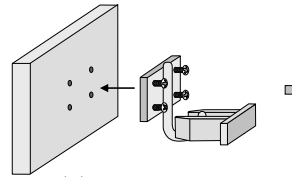

(g)

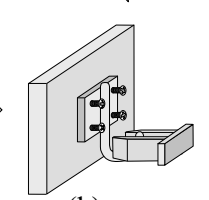

(h)

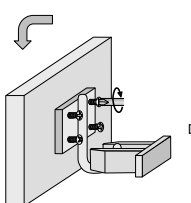

(i)

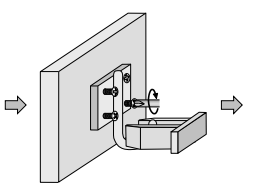

(j)

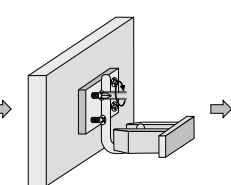

(k)

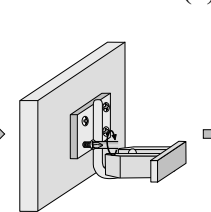

(1) $(\mathrm{m})$

Fig. 10 [ER-II] Error Recovery II

The amount of data set is important when using a data science method. Even if the execution of operation is extremely difficult, a considerable amount of data enables the work to be performed skillfully.

(vii) Tool

Priority order: A process with an exclusive tool is given priority.

If a special tool for recovery is available, there is a high possibility that the process surely returns in a short time. If there is a tool by which four screws can be fastened temporarily as for any posture of a target, [ER-III(N)] (or [ER-III(F)]) becomes a top-priority process.

(viii) Operator skill

Priority order: A process of forte of teaching operator is given priority.

The recovery process in which the teaching operator is used is the most suitable. There is a possibility that a suitable process for an expert operator is different from that of an unskilled operator.

(C) The 2021 International Conference on Artificial Life and Robotics (ICAROB2021), January 21 to 24, 2021 


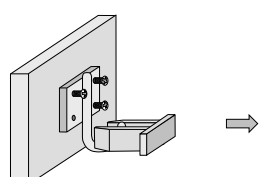

(h)'

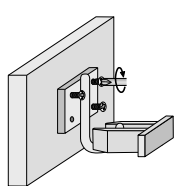

(i)

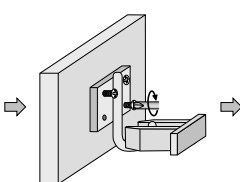

(j)

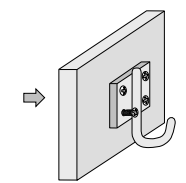

(v)

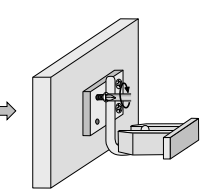

(k)

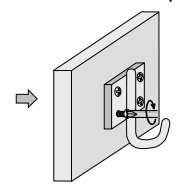

(1)

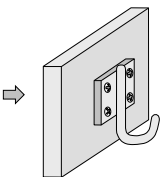

(m)

Fig. 11 [ER-III] Error Recovery III

We considered a suitable process derived by selecting one evaluation standard at a time from eight. However, in actuality, there is a possibility that the optimum process is derived using multiple evaluation standards.

\section{Conclusion}

When an error occurs in automated plants, the process advances to the recovery part. In general, many types of recovery processes can be considered. In this study, we have shown a method to derive a suitable process using only one evaluation standard at a time selected from various standards. The preferential order of recovery processes can be obtained considering each evaluation standard.

However, we have used only one standard, although we did consider eight types of evaluation standards. Therefore, in future studies, we will include techniques to derive a suitable recovery process using several evaluation standards simultaneously and determine the applicability of our method to actual systems.

\section{Acknowledgements}

This work was supported by New Energy and Industrial Technology Development Organization (NEDO) in Japan.

\section{References}

1. B. R. Donald, Planning multi-step error detection and recovery strategies, Int. J. Robot. Res., 9(1) (1990) 3-60.

2. T. Niemueller, G. Lakemeyer and S. S. Srinivasa, A Generic Robot Database and its Application in Fault Analysis and Performance Evaluation, in Proc. IEEE/RSJ Int. Conf. on Intell. Robots Syst., (Vilamoura, Portugal, 2012), 364-369.
3. E. D. Lello, M. Klotzbucher, T. D. Laet and H. Bruyninckx, Bayesian Time-Series Models for Continuous Fault Detection and Recognition in Industrial Robotics Tasks, in Proc. IEEE/RSJ Int. Conf. on Intell. Robots Syst., (Tokyo, Japan, 2013), 5827-5833.

4. E. Krabbe, E. Kristiansen, L. Hansen and D. Bourne, Autonomous Optimization of Fine Motions for Robotic Assembly, in Proc. IEEE Int. Conf. Robot. Autom., (Hong Kong, China, 2014), 4168-4175.

5. A. S. Wang and O. Kroemer, Learning Robust Manipulation Strategies with Multimodal State Transition Models and Recovery Heuristics, in Proc. IEEE Int. Conf. Robot. Autom., (Montreal, Canada, 2019), 1309-1315.

6. A. Nakamura, K. Nagata, K. Harada, N. Yamanobe, T. Tsuji, T. Foissotte and Y. Kawai, Error recovery using task stratification and error classification for manipulation robots in various fields, in Proc IEEE/RSJ Int. Conf. on Intell. Robots Syst., (Tokyo, Japan, 2013), 3535-3542.

7. A. Nakamura, K. Nagata, K. Harada and N. Yamanobe, Technique of Recovery Process and Application of AI in Error Recovery Using Task Stratification and Error Classification, J. Robotics, Networking and Artificial Life, 5(1), 2018, pp. 56-62.

8. A. Nakamura, N. Yamanobe, I. R. Alpizar, K. Harada and Y. Domae, Cost-oriented Planning for Error Recovery in an Automation Plant, J. Robotics, Networking and Artificial Life, 6(4), 2020, pp. 225-230.

9. T. Hasegawa, T. Suehiro and K, Takase, A robot system for unstructured environments based on an environment model and manipulation skills, in Proc. IEEE Int. Conf. Robot. Autom., (Sacramento, USA, 1991), 916-923.

10. A. Nakamura, T. Ogasawara, T. Suehiro and H. Tsukune, Fine motion strategy for skill-based manipulation, Artificial Life and Robotics, Springer, 1(3), 1997, pp 147-150.

11. A. Nakamura, K. Kitagaki and T. Suehiro, Using simplified geometric models in skill-based manipulation, Advanced Robotics, 18(8), 2004, pp. 835-858. 